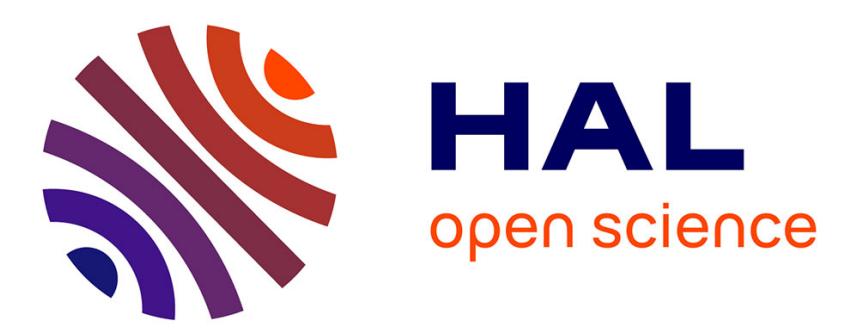

\title{
Three-dimensional numerical modelling of gas discharges at atmospheric pressure incorporating photoionization phenomena
}

\author{
L Papageorgiou, a C Metaxas, G E Georghiou
}

\section{To cite this version:}

L Papageorgiou, a C Metaxas, G E Georghiou. Three-dimensional numerical modelling of gas discharges at atmospheric pressure incorporating photoionization phenomena. Journal of Physics D: Applied Physics, 2011, 44 (4), pp.45203. 10.1088/0022-3727/44/4/045203 . hal-00589769

\author{
HAL Id: hal-00589769 \\ https://hal.science/hal-00589769
}

Submitted on 2 May 2011

HAL is a multi-disciplinary open access archive for the deposit and dissemination of scientific research documents, whether they are published or not. The documents may come from teaching and research institutions in France or abroad, or from public or private research centers.
L'archive ouverte pluridisciplinaire HAL, est destinée au dépôt et à la diffusion de documents scientifiques de niveau recherche, publiés ou non, émanant des établissements d'enseignement et de recherche français ou étrangers, des laboratoires publics ou privés. 


\title{
Three-dimensional numerical modelling of gas discharges at atmospheric pressure incorporating photoionisation phenomena
}

\author{
L. Papageorgiou ${ }^{1}$, A. C. Metaxas ${ }^{2}$ and G. E. Georghiou ${ }^{1}$ \\ ${ }^{1}$ Department of Electrical and Computer Engineering, University of Cyprus, 75 Kallipoleos Avenue, P.O. \\ Box 20537 Nicosia, 1678, Cyprus \\ ${ }^{2}$ St John's College, University of Cambridge, Cambridge CB2 1TP, U.K
}

\begin{abstract}
A three-dimensional (3D) numerical model for the characterization of gas discharges in air at atmospheric pressure incorporating photoionisation through the solution of the Helmholtz equation is presented. Initially, comparisons with a two-dimensional (2D) axi-symmetric model are performed in order to assess the validity of the model. Subsequently several discharge instabilities (plasma spots and low pressure inhomogeneities) are considered in order to study their effect on streamer branching and off-axis propagation. Depending on the magnitude and position of the plasma spot, deformations and off-axis propagation of the main discharge channel were obtained. No tendency for branching in small (of the order of $0.1 \mathrm{~cm}$ ) overvolted discharge gaps was observed.
\end{abstract}

\section{Introduction}

Gas discharges at atmospheric pressure have been attracting a lot of attention recently due to the inherent advantages they offer in producing plasma, namely low cost production without the need for costly enclosures. Plasma medicine [1] and plasma actuators [2] are two areas where these discharges are currently being used widely. Due to their nature, the experimental characterization of such discharges is very difficult: their temporal evolution is of the order of a few nanoseconds while their spatial resolution is in the range of a few micrometers. Additionally, during their formation and propagation strong non-linear phenomena take place making their characterization even more challenging.

A common phenomenon observed in atmospheric pressure gas discharges in air is streamers; these are ionisation waves that propagate with velocities much higher than the maximum drift velocity of electrons. Their spatial structure is inherently three-dimensional; their experimental investigation is very difficult rendering numerical modelling a very important tool in determining a proper understanding of streamer physics $[3,4]$. Until now, numerous papers have been published involving one-dimensional (1D) and two-dimensional (2D) streamer modelling, elucidating several characteristics of the streamer nature [5]. Two-dimensional axi-symmetric models are predominantly employed allowing the study of some phenomena in a three-dimensional setting as well. Work based on 1.5D and 2D models [6-8] has been conducted by making several assumptions in an attempt to understand several aspects of streamer branching and streamer interaction. However, one important drawback of the 2D axi-symmetric models is that they restrict gas discharge modelling in configurations and conditions that are inherently symmetric around the axis of symmetry.

Phenomena like (a) streamer branching, (b) electrode configurations with no cylindrical symmetry, (c) interaction between streamers and (d) non homogeneous surfaces, which have inherently a threedimensional character, cannot be dealt with in a 2D axi-symmetric setting. Complete numerical characterization has to be based on fully three-dimensional models such as the ones developed by Hallac et al [9], Pancheshnyi [10], Luque [11] and Kulikovsky [12]. The 3D models of Luque [11] and Kulikovsky [12] are axi-symmetric with an expansion of the $\varphi$ coordinate around the axis of symmetry. Questions on resolution and subsequently accuracy in the $\varphi$ direction arise in regions away from the axis of symmetry in the radial direction. In the work of Hallac et al [9] photoionisation was 
introduced via a truncated implicit subgridding method. Pancheshnyi et al [10] presented results from an efficient parallel fully 3D streamer model but without the incorporation of photoionisation. Here we make a contribution by fully incorporating photoionisation in order to provide the whole picture during the formation and propagation of the streamer. Photoionisation phenomena are taken into account by solving a set of Helmholtz equations as suggested by Luque et al [13] and Bourdon et al [14] (this is in contrast to the computationally demanding integral method developed by Zeleznyak et al [15], introduced in 2D models by Kulikovsky [16]).

The approach proposed by Luque et al [13] is mainly based on fitting the absorption function of photo-ionization radiation with a sum of exponential functions leading to a set of integrals, each of which can effectively be interpreted as an integral solution of a separate Helmholtz differential equation. After this equivalent representation is established the integral problem of estimating the photoionization can be obtained solved by solving the set of Helmholtz differential equations, instead of direct directly evaluating the of integrals [14]. In our case, photoionization occurs throughout absorption by oxygen molecules, of the radiation emitted by nitrogen molecules in singlet excited states $b^{1} \Pi, b^{1} \Sigma_{u}{ }^{+}$and $c_{4}{ }^{1} \Sigma_{\mathrm{u}}{ }^{+}$in the wavelength range $98-102.5 \mathrm{~nm}[17]$.

In this work a preliminary examination on how several discharge instabilities (plasma spots and low pressure inhomogeneities) could affect streamer branching and off axis propagation is conducted. For such studies a fully 3D numerical scheme is required. First we validate our new 3D numerical scheme with a $2 \mathrm{D}$ axi-symmetric one by considering a situation were both schemes are equally valid. After the validation phase, the three dimensional model can be used with confidence to study the problems stated above.

Plasma spots can arise by irradiation of the gap with UV laser pulses; for example, whereas low pressure inhomogeneities represent fluctuations in the pressure that could arise in the gap. The study of such parameters is very instructive in the further understanding of streamer branching and gas discharge development. The results demonstrate clearly that, depending on the magnitude and position of the plasma spot, streamer head deformation and off axis propagation of the discharge channel are observed. On the other hand no tendency for branching in small (of the order $0.1 \mathrm{~cm}$ ) overvolted $\begin{array}{llll}\text { discharge gaps has observed. } & \text { been }\end{array}$

\section{Governing equations}

The differential equations that describe the cold plasma are the Poisson equation for the electric field, the continuity equations for charged particles and the Helmholtz equation for the photoionisation phenomenon. These are shown below:

$$
\begin{aligned}
& -\nabla \cdot \varepsilon \nabla \Phi-\sum_{j} N_{j} q_{j}+r_{S}=0 \\
& \frac{\partial N_{e}}{\partial t}+\nabla \cdot\left(N_{e} \vec{W}_{e}\right)-\nabla \cdot\left(D_{e} \nabla N_{e}\right)=S_{e} \\
& \frac{\partial N_{i}}{\partial t}+\nabla \cdot\left(N_{i} \vec{W}_{i}\right)=S_{i} \\
& \frac{\partial N_{n}}{\partial t}+\nabla \cdot\left(N_{n} \vec{W}_{n}\right)=S_{n} \\
& S_{e}=\alpha N_{e}\left|\vec{W}_{e}\right|-\eta N_{e}\left|\overrightarrow{W_{e}}\right|-\beta_{e p} N_{e} N_{p}+S_{p h} \\
& S_{i}=\alpha N_{e}\left|\vec{W}_{e}\right|-\beta_{e p} N_{e} N_{p}-\beta_{p n} N_{p} N_{n}+S_{p h} \\
& S_{n}=\eta N_{e}\left|\vec{W}_{e}\right|-\beta_{p n} N_{p} N_{n} \\
& \nabla^{2} S_{p h}-\left(\lambda p_{O_{2}}\right)^{2} S_{p h}=-A p_{O_{2}}^{2} I
\end{aligned}
$$


where $t$ is the time, $N_{\mathrm{e}}, N_{\mathrm{p}}$ and $N_{\mathrm{n}}$ are the charge densities for electrons, positive ions and negative ions respectively. $\vec{W}_{e}, \vec{W}_{p}$ and $\vec{W}_{n}$ represent the drift velocities for electrons, positive ions and negative ions respectively and $D_{e}$ is the electron diffusion coefficient. The symbols $\alpha, \eta, \beta_{\text {ep }}$ and $\beta_{\text {np }}$ denote the ionisation, attachment, electron-positive-ion recombination and negative-ion-positive-ion recombination coefficients respectively. All of these quantities, with the exception of recombination coefficients, need to be known as a function of the electric field. In the present model the negative ions are considered to have negligible influence on the timescales considered for this problem. $\Phi$ is the electric potential, $n_{j} q_{j}$ the densities of the charged species and $r_{s}$ the surface charge. $S_{p h}$ is the source term due to photoionisation, $p_{\mathrm{O}_{2}}$ is the partial pressure of oxygen and $I$ is the emission of photons in the discharge volume assumed to be proportional to the number of ionizing collisions which are given by the product $a \cdot n_{e} \cdot \vec{W}_{e} \cdot \lambda$ and $A$ are fitting parameters [14]. The parameters for the two-exponential fit are presented in the table 1 .

Table 1: Parameters of the two exponential fit

\begin{tabular}{|l|c|c|}
\hline & $A\left(\mathrm{~cm}^{-2}\right.$ Torr $\left.^{-2}\right)$ & $\lambda\left(\mathrm{cm}^{-1}\right.$ Torr $\left.^{1}\right)$ \\
\hline 1 & 0.0021 & 0.0974 \\
\hline 2 & 0.1775 & 0.5877 \\
\hline
\end{tabular}

The computational cost for 3D gas discharge modelling is very high. This means that special attention should be paid in choosing the mathematical relations that yield the transport coefficients. These relations have to be as mathematically simple as possible without loss of accuracy. For this reason, the transport coefficients by Kang et al [18] and the drift velocity by Morrow and Lowke [19] have been used.

The boundary conditions of the 3D model are shown below (see Table 2):

Table 2: Boundary conditions used

\begin{tabular}{|l|l|l|l|}
\hline & Electrons & Ions & Voltage \\
\hline Anode & $\vec{n} \cdot\left(D_{e} \cdot \nabla N_{e}\right)=0$ & $N_{i}=1000 \mathrm{~cm}^{-3}$ & $5000 \mathrm{~V}$ \\
\hline Cathode & $J_{e}=\gamma \cdot N_{i} v_{i}$ & $\vec{n} \cdot\left(D_{i} \cdot \nabla N_{i}\right)=0$ & $0 \mathrm{~V}$ \\
\hline
\end{tabular}

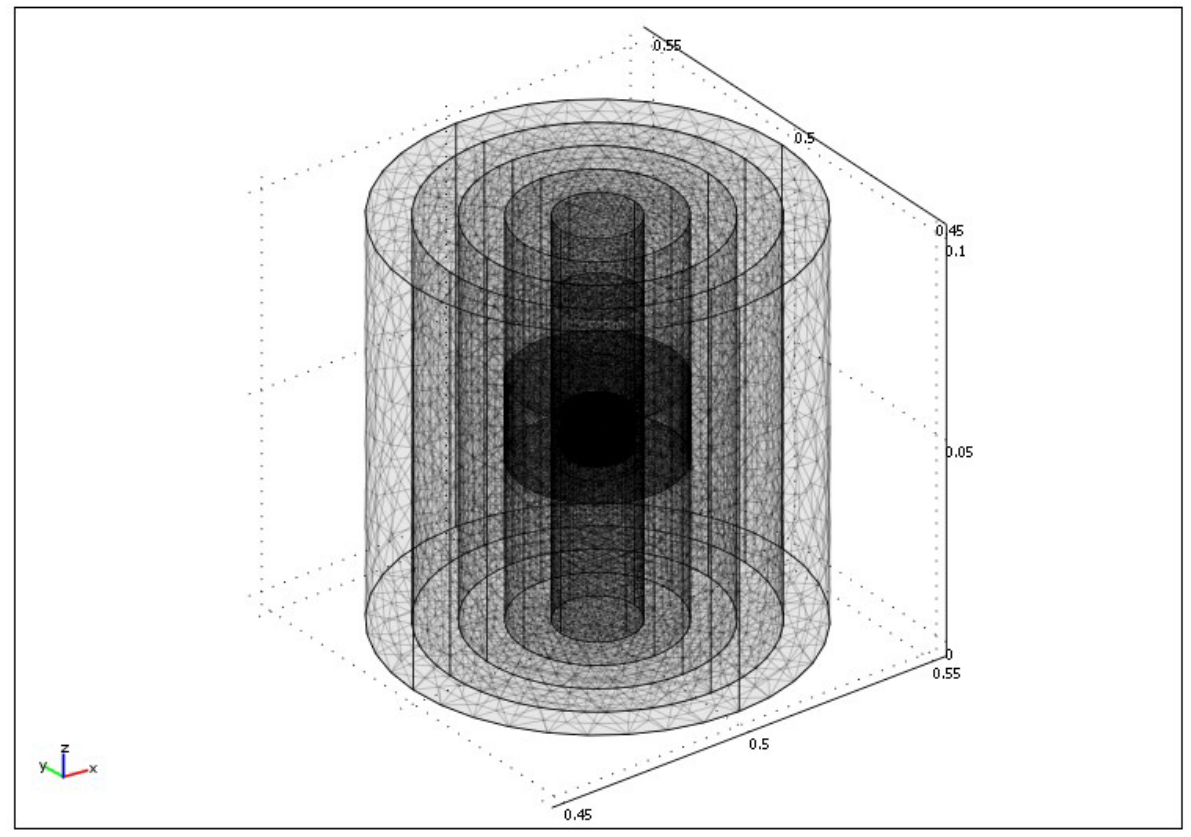

a) Mesh used for the 3D simulations 


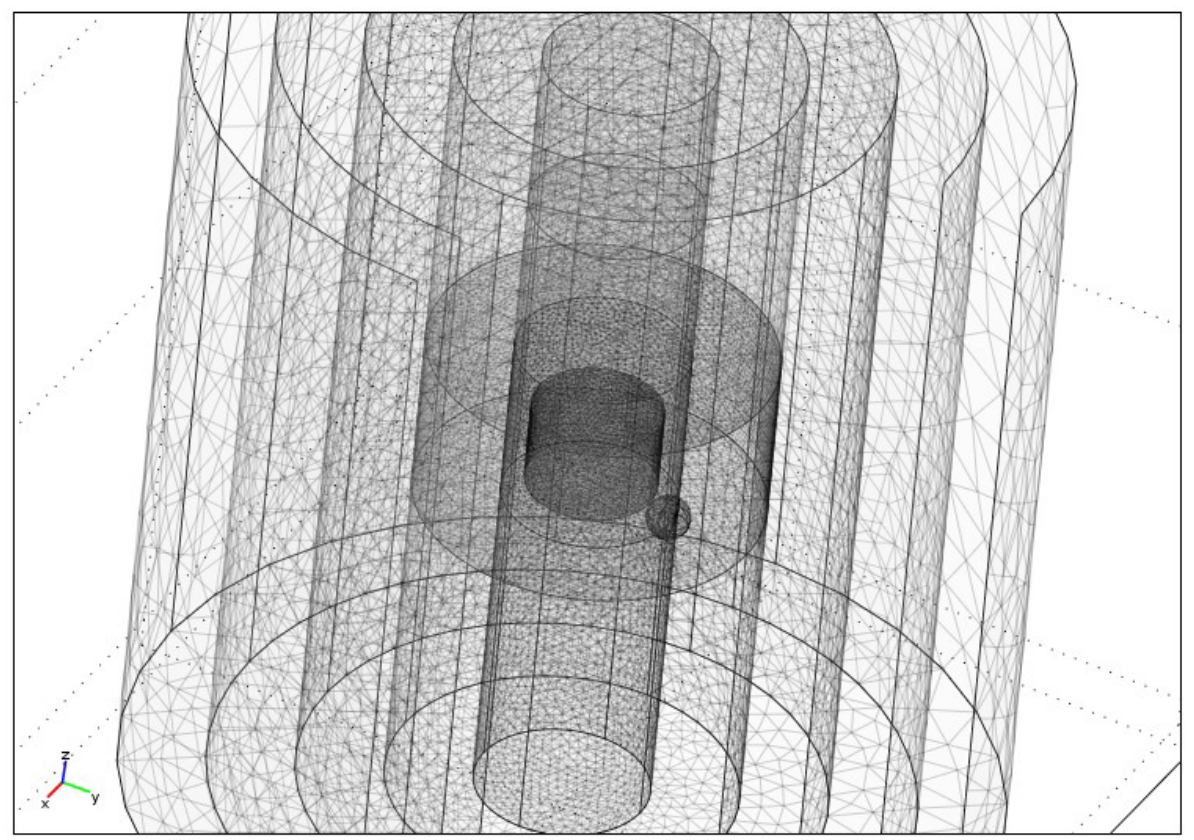

b) Closer look of the mesh used for the 3D simulations

Figure 1. Three-dimensional mesh used for streamer propagation. The mesh is gradually becoming finer following an onion-like pattern as approaching the axis of the discharge.

At the anode the electrons are convected freely through the metallic electrode and the ions show the same behaviour at the cathode. Secondary electron emission from the cathode due to ion bombardment is taken into account by setting the gamma coefficient equal to 0.01 . For the Helmholtz equations (8) Dirichlet boundary conditions equal to zero are introduced at the boundaries of the computational region.

\section{Results}

In this paper a plane to plane electrode configuration with a gap size of $0.1 \mathrm{~cm}$ is considered. An initial Gaussian electron and positive ion density (plasma spot $n\left(\mathrm{~cm}^{-3}\right)$ given by the expression below) with maximum value $A=10^{10} \mathrm{~cm}^{-3}$ and width $\sigma_{o}=0.005 \mathrm{~cm}$ (9) are introduced in the gap at the cathode and a voltage of $5000 \mathrm{~V}$ is applied in the inter-electrode space.

$n=A \cdot\left(e^{\frac{-\left(x-x_{0}\right)^{2}}{\left(2 \cdot \sigma_{0}{ }^{2}\right)}+\frac{-\left(y-y_{0}\right)^{2}}{\left(2 \cdot \sigma_{0}{ }^{2}\right)}+\frac{-\left(z-z_{0}\right)^{2}}{\left(2 \cdot \sigma_{0}{ }^{2}\right)}}\right)$

An unstructured mesh, as shown in Figure 1, was employed and optimized in order to reduce as much as possible the computational cost. The mesh is gradually becoming finer following an onion-like pattern as approaching the axis of the discharge. For the Poisson and continuity equations Lagrangequadratic elements were used while for the Helmholtz equations Lagrange linear elements were employed. The solver of the model is based on the Finite Element Method and uses Newton's iterations for the solution of the non-linear system of equations. With that approach we demand the whole system of equations to converge simultaneously. The time stepping algorithm is based on a fifth order Backward Differentiation Formula (BDF) which is an implicit method for the numerical integration the differential equations. The adopted numerical techniques allow the use of relatively large time-steps not restricted by the Courant-Friedrichs-Lewy (CFL) condition. The CFL condition slows down most of the high resolution schemes which are used in streamer modelling for the solution 
of the hyperbolic continuity equations. The evolution of the discharge reveals two well known phases: the avalanche and the streamer propagation phase.

\subsection{Streamer evolution}

The initial electrons under the influence of the electric field accelerate and multiply via ionisation processes as presented in Figure 2. The deformation of the initial Gaussian shape is apparent close to the anode where space charge effects become important. In Figure 3, the electron density close to the anode is depicted. The velocity of the avalanche is about $2.3 \times 10^{7} \mathrm{~cm} / \mathrm{s}$. This value is in accordance with results from $2 \mathrm{D}$ models, revealing that no phase error is introduced in the results during this part of the discharge.

The streamer formation and propagation phase is much more computationally demanding than the avalanche one. The density gradients at the streamer head are very steep and the phenomenon advances with higher velocities. For this reason a moving window of high mesh resolution is set to follow the streamer head movement. The size of the mesh that gives accurate results is of the order of $5 \mu \mathrm{m}$. In Figure 4 the evolution of the electron density on the axis of symmetry of the discharge is shown.

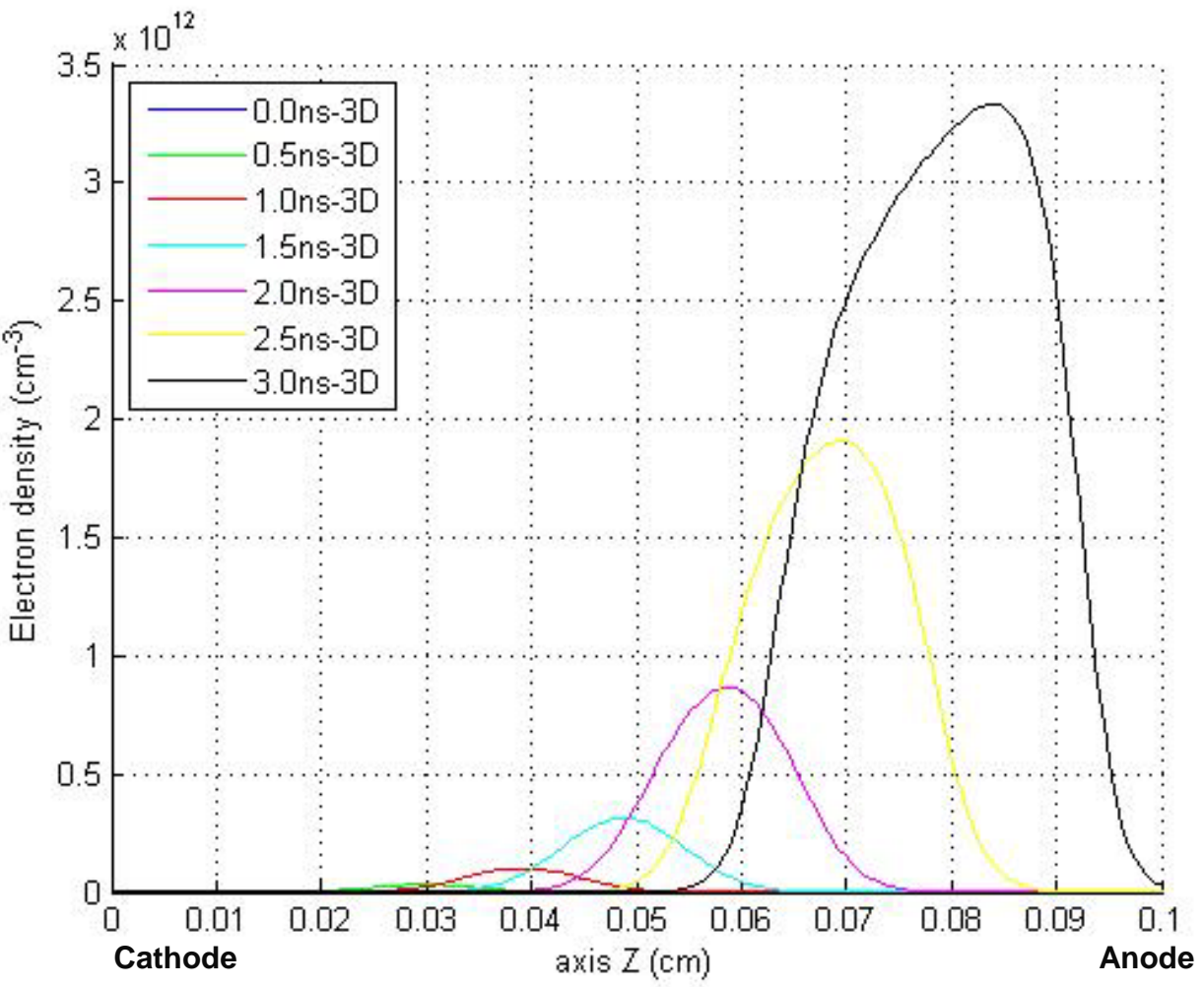

Figure. 2 Electron density profile evolution on the axis of symmetry during the avalanche phase 
Time $=3 e^{-9} \quad$ Slice: Concentration, ne

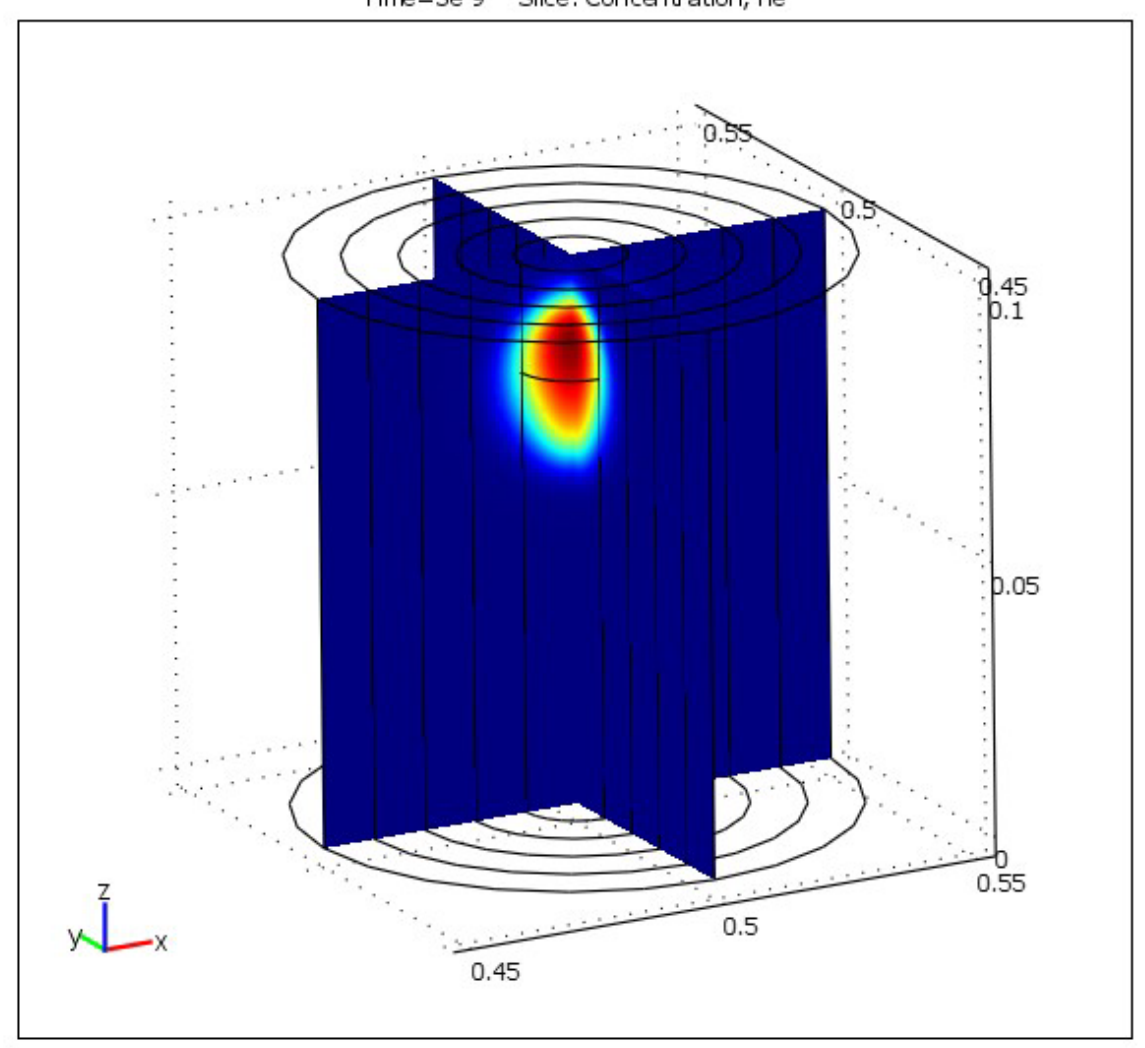

Max: $3.326 \mathrm{e} 12$

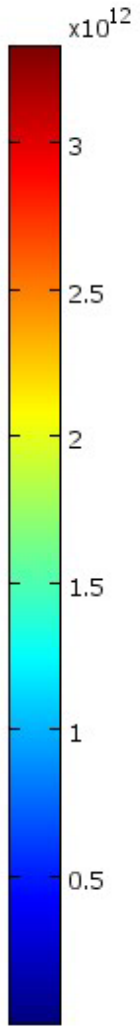

Min: 135.804

Figure. 3 Three-dimensional electron density $\left(\mathrm{cm}^{-3}\right)$ profile at $3.0 \mathrm{~ns}$

The results are monotonic and positive. In figure 5 the evolution of the electric field on the axis of symmetry is also presented. The electric field magnitude at the head of the streamer has a value of $200 \mathrm{kV} / \mathrm{cm}$ whereas in the ionized channel behind the propagating front towards the cathode the value is approximately $25 \mathrm{kV} / \mathrm{cm}$. It is observed that the shielding in the quasi-neutral channel behind the streamer head is of the order of 50\% compared to the geometric field. Similar values for the electric field in the plateau of the discharge have been also been observed in [20]. In Figure 6 the electron density when the streamer is in the middle of the gap is presented. The discharge channel that is formed has a radius of approximately $50 \mu \mathrm{m}$, a value that is closely dependant on the choice of photoionization radiation model parameters [20], and on the width $\left(\sigma_{0}\right)$ of the initial Gaussian plasma spot. For $\sigma_{0}=25 \mu \mathrm{m}$ a much narrower streamer was observed in accordance with the results presented in [21].

The streamer velocity and diameter increase as the streamer approaches the cathode. This behavior agrees with already published 2D results [22] but deviates from experimental observations that show streamer propagation with a constant channel radius in plane to plane gaps [23]. This difference is attributed to the longer gap employed for the experimental observations allowing the streamer radius to reach a steady state value. 


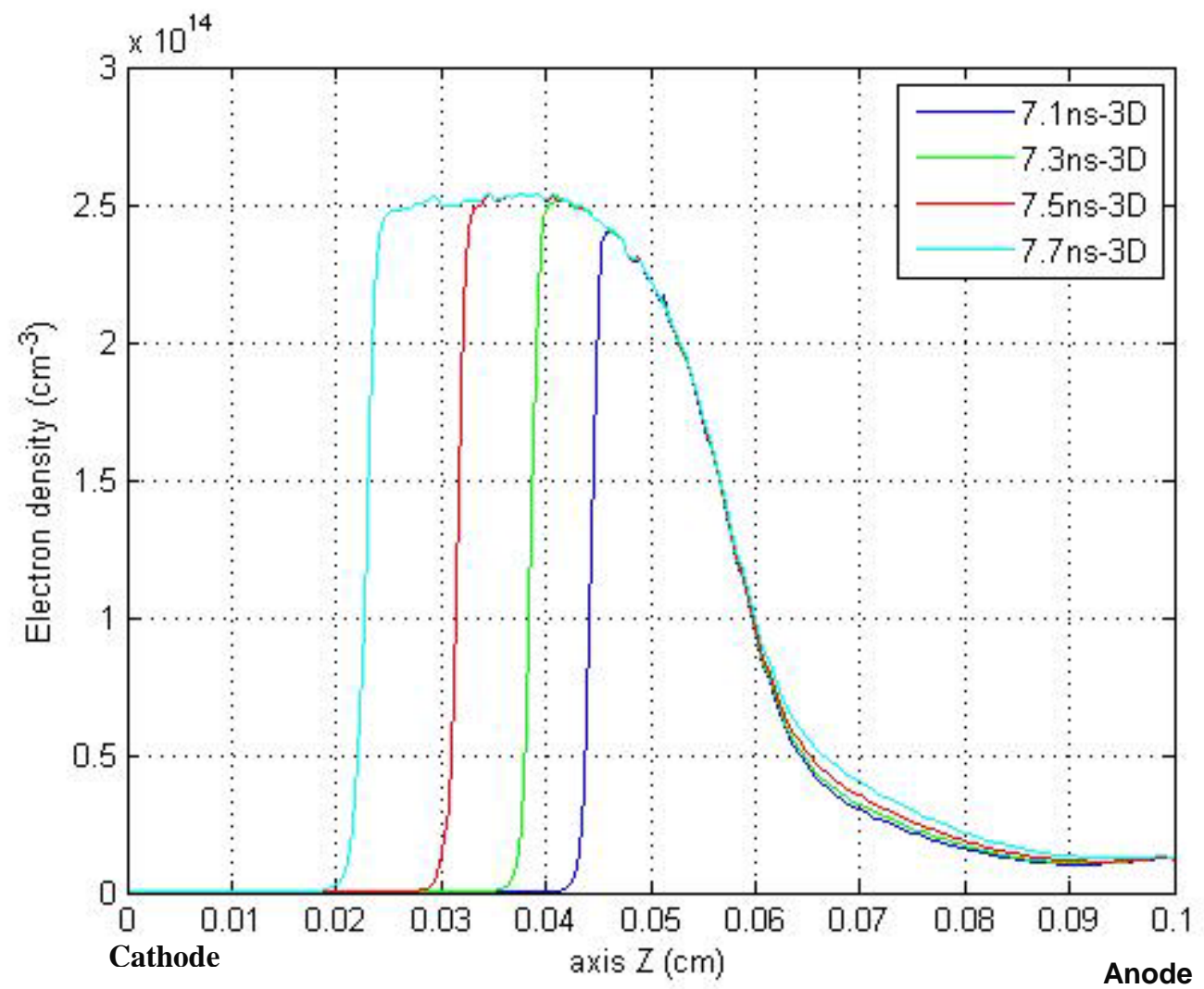

Figure. 4 Evolution of the electron density profile on the axis of symmetry during the streamer phase

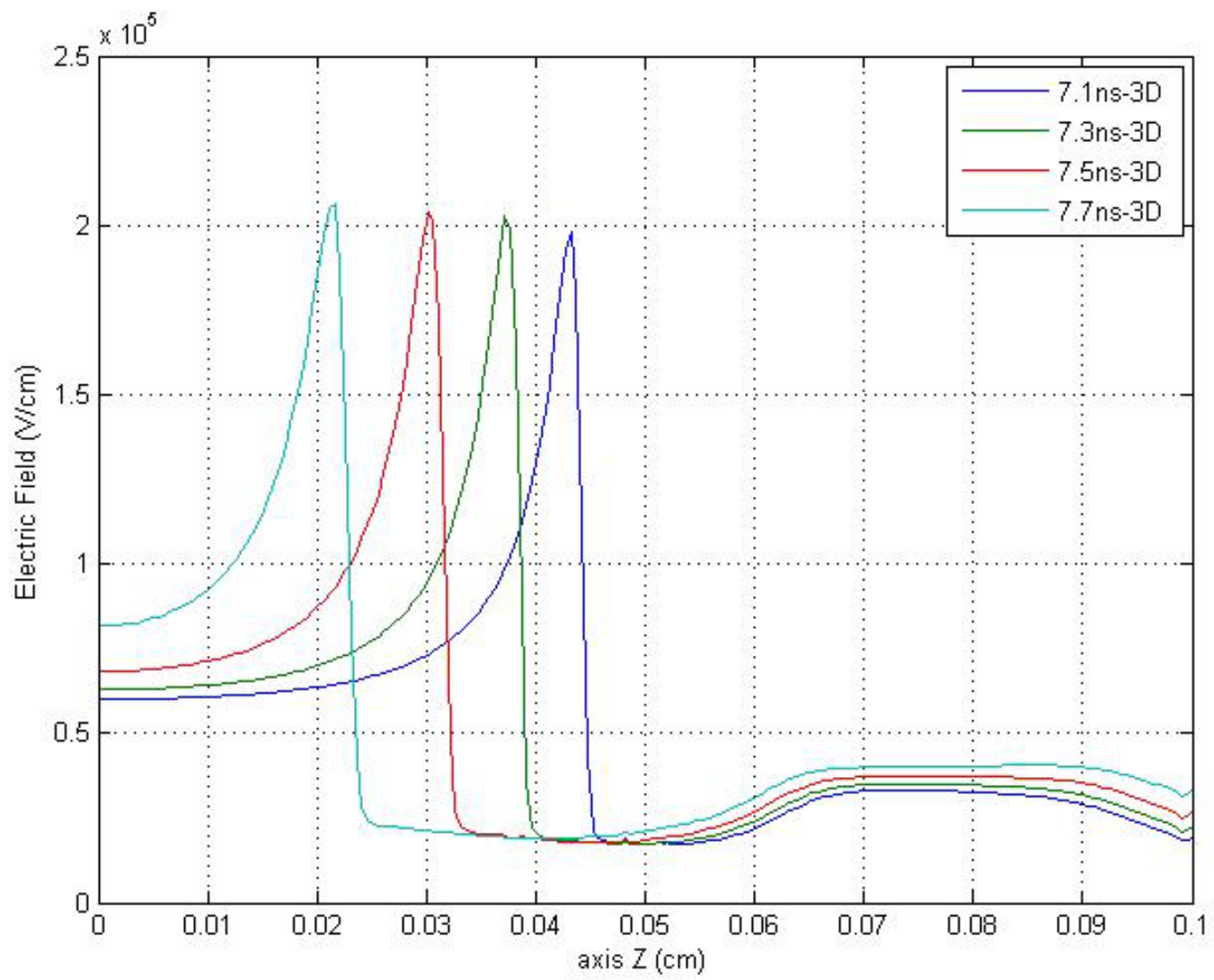

Cathode

Anode

Figure. 5 Evolution of the electric field profile on the axis of symmetry during the streamer phase 

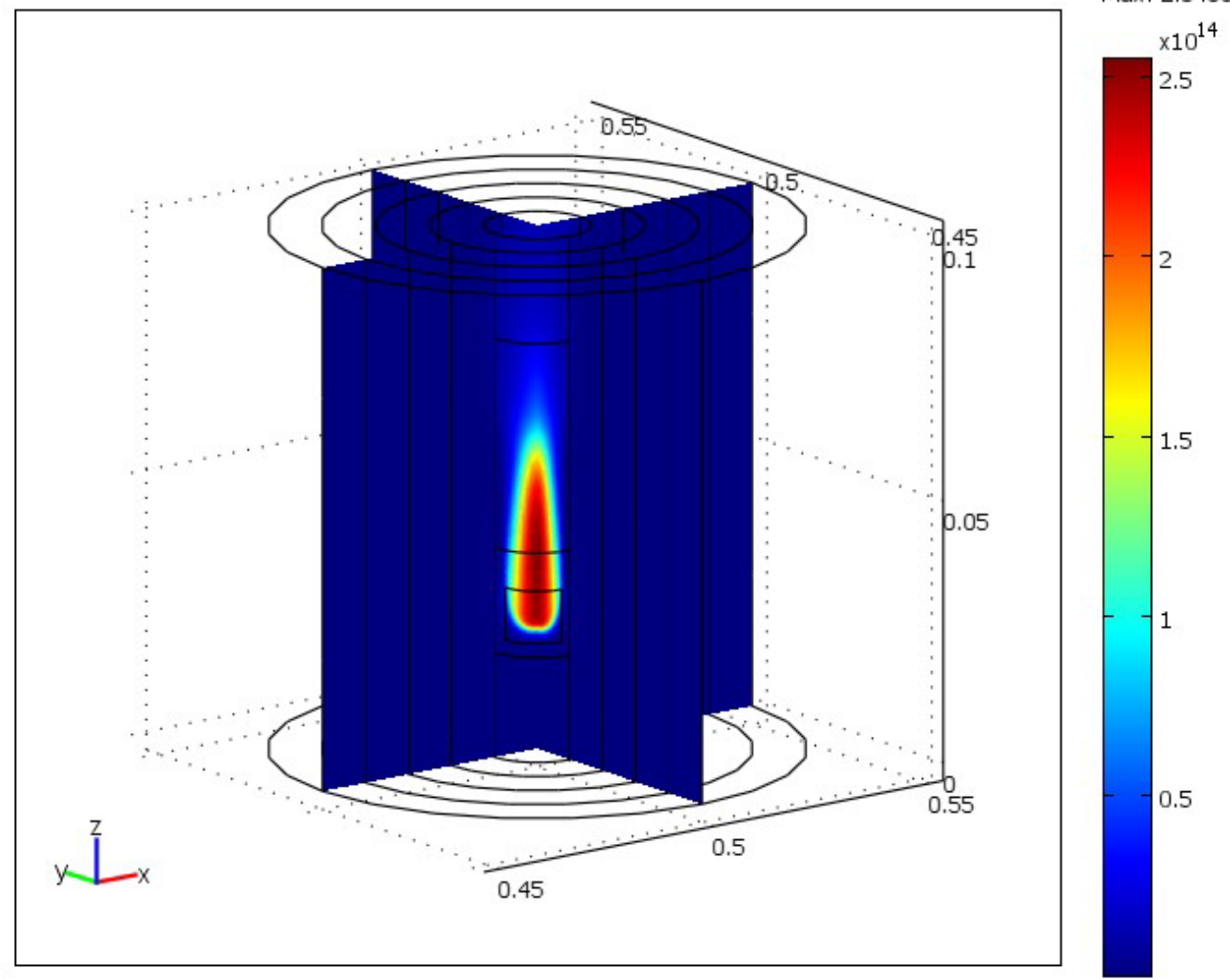

Min: $4.163 e 5$

Figure. 6 Three-dimensional electron density $\left(\mathrm{cm}^{-3}\right)$ profile at 7.9 ns during streamer propagation

\subsection{Comparison of 2D axi-symmetric and 3D models}

The comparison between $2 \mathrm{D}$ axi-symmetric and $3 \mathrm{D}$ results is very instructive as it will first of all provide confidence in the validity of the newly developed 3D model. In Figure 7 the initial photoionisation radiation from both models is shown. The solution of the 2D axi-symmetric and the 3D model gives almost identical results. It is worth noting that the three-exponential Helmholtz photoionisation model [14] was also implemented giving a very similar discharge evolution. In our work the two-exponential model was finally adopted in order to reduce the computational cost. In Figures 8 and 9, the electron density profiles on the axis of symmetry of the discharge during the avalanche and streamer phases for the two different models (2D and 3D) are presented. In Figure 10 the current density calculated by Sato's formula [24] for the 2D and 3D model is also provided yielding very good agreement. 


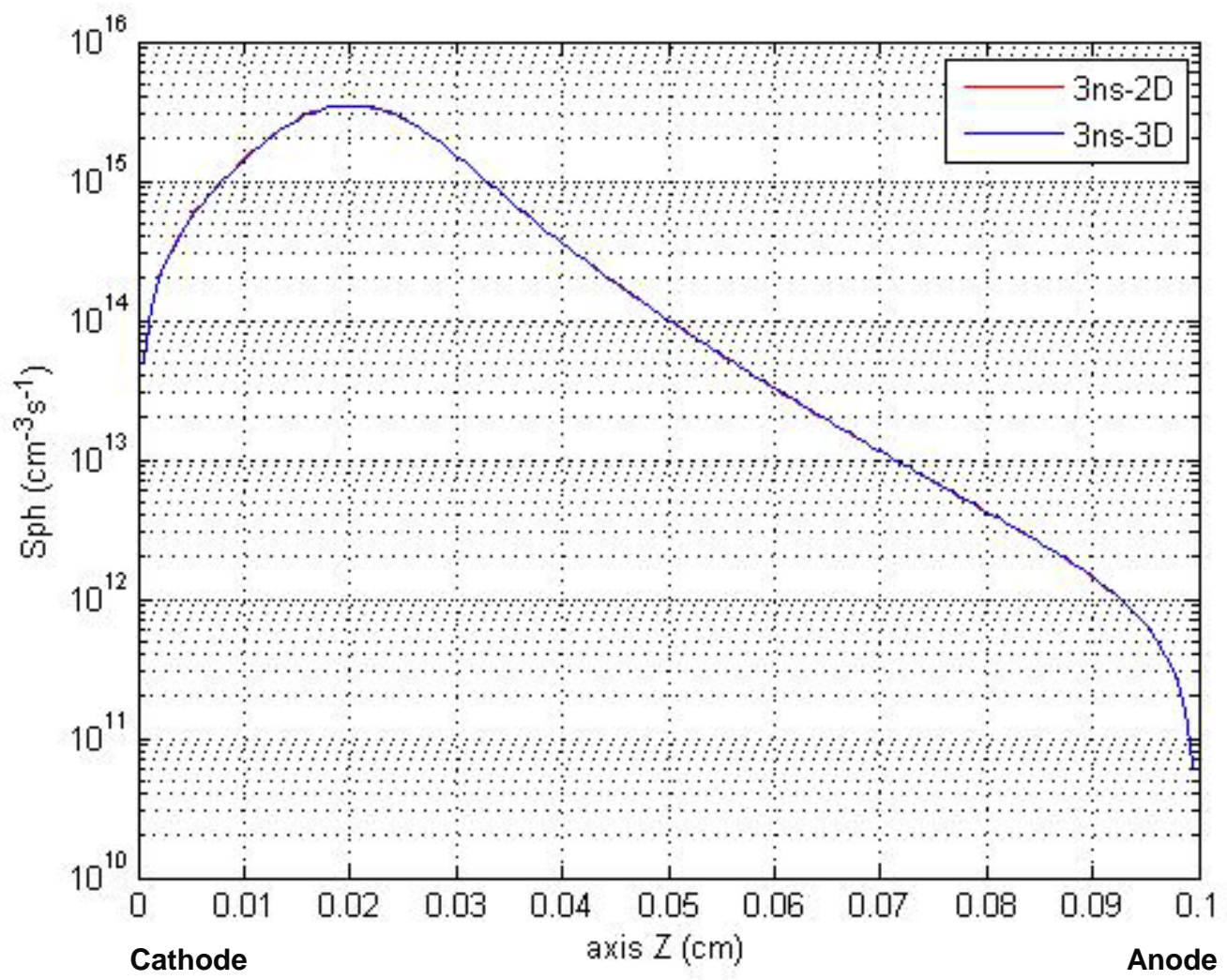

Figure. 7 Initial photoionisation radiation on the axis of symmetry for the 2D (red line) and 3D (blue line) model

The comparison is performed for exactly the same external conditions and mesh resolution. During the avalanche phase the resolution is set to $15 \mu \mathrm{m}$ whereas during the streamer phase a moving window consisting of elements of size $5 \mu \mathrm{m}$ follows the streamer head. At the avalanche and streamer phases the comparison between the $2 \mathrm{D}$ axi-symmetric and fully $3 \mathrm{D}$ results yield very good agreement (see Figures 8 and 9). This is in disagreement with the results in [10] where it is claimed that the 3D model gives more diffusive results compared to the 2D ones. In Figure 8 there are no differences in the density profiles whereas in Figure 9, during the streamer phase, the density profile of the 3D model is slightly ahead of the corresponding $2 \mathrm{D}$ profile. This difference is due to interpolation errors that are introduced in the 3D solution every time the moving window is updated to follow the streamer head. The discharge current (Figure. 10) for both cases is almost identical. The hump observed at about $3 \mathrm{~ns}$ is due to the absorption of the initial electron distribution at the anode (see Morrow and Lowke Figure $1[18])$. 


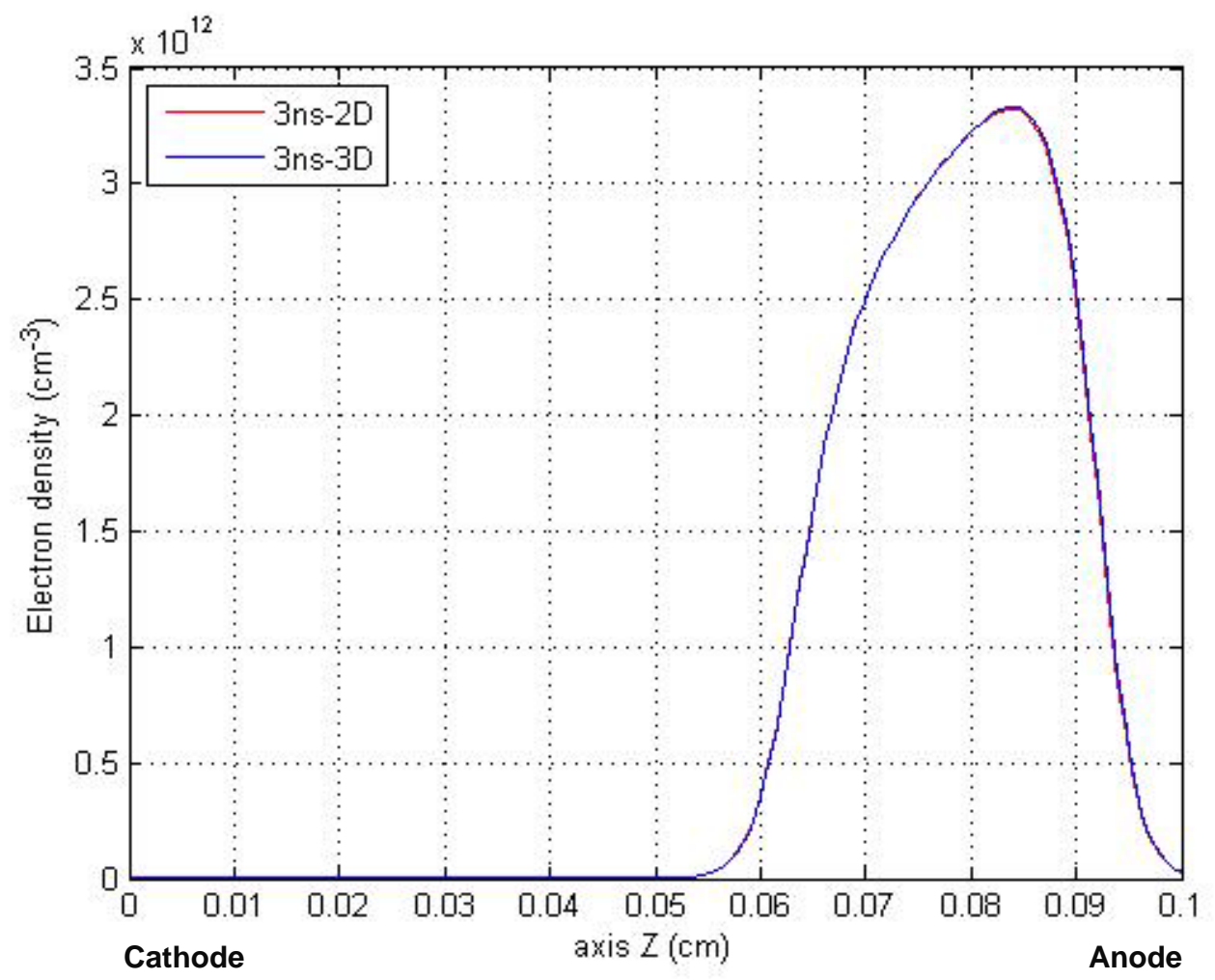

Figure. 8 Comparison of the electron density profiles on the axis of symmetry between the 2D (red line) and 3D (blue line) model during the avalanche phase at $3.0 \mathrm{~ns}$.

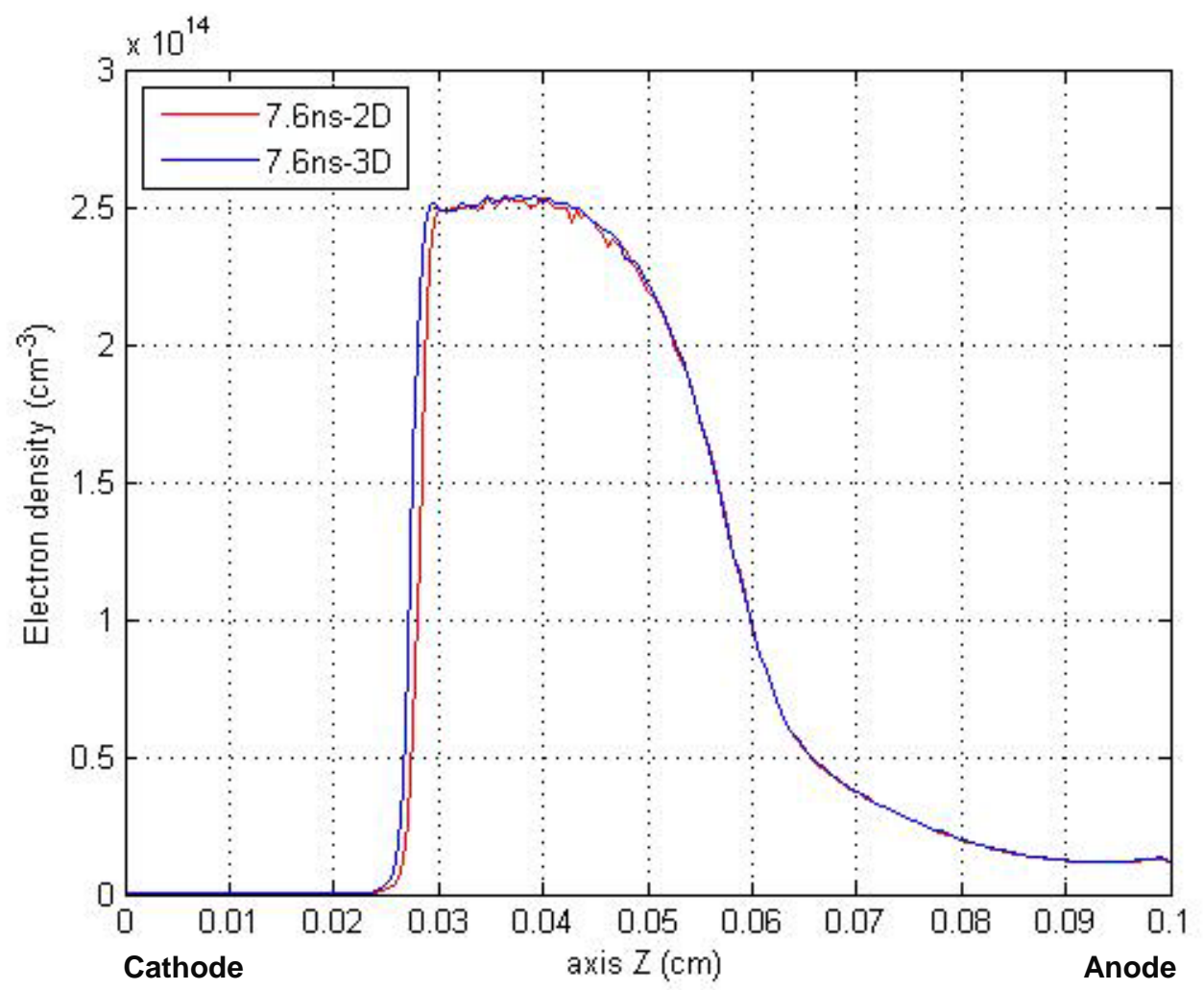

Figure. 9 Comparison of the electron density profiles on the axis of symmetry between the 2D (red line) and 3D (blue line) model during the streamer phase at $7.6 \mathrm{~ns}$. 
Another crucial parameter in gas discharge numerical modelling and especially in 3D problems is the computational cost (see Table 3). The simulation time for the discharge evolution in $2 \mathrm{D}$ was approximately 20 minutes, whereas in $3 \mathrm{D}$ the calculation takes one week. In $2 \mathrm{D}$ the degrees of freedom (DoFs) are of the order of 36,000 while in 3D, when the streamer head is at the middle of the gap the resolution has to be very high, of the order of 4,000,000. The need for RAM in 3D is also very high and approximately $15 \mathrm{~GB}$ in this case. This shows clearly that 2D models have to be adopted in cases where the cylindrical symmetry is preserved and especially in large gaps; the computational cost of the 3D models is very high. The above simulations were performed on a system with four Intel Xeon 5500 series cores.

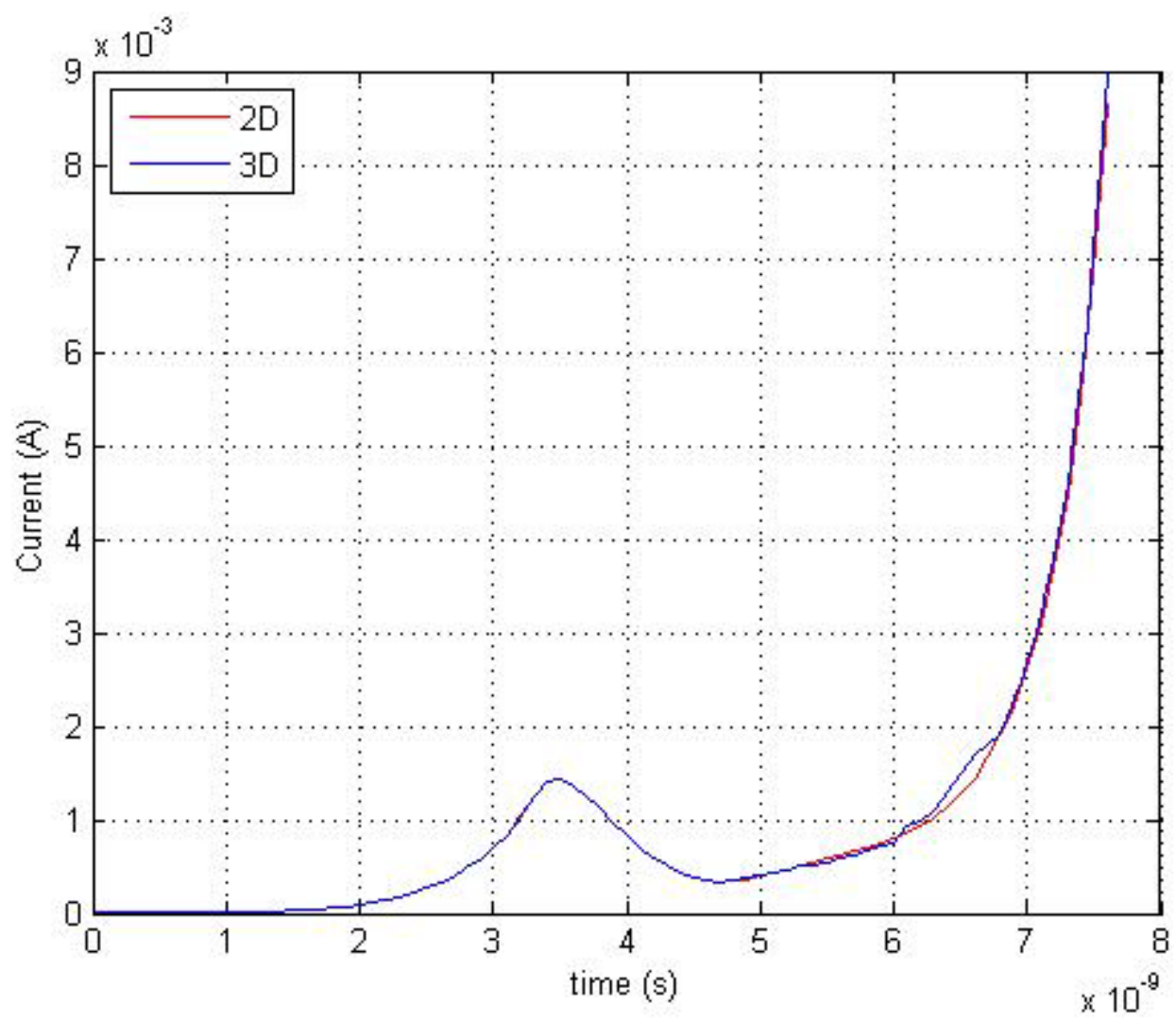

Figure. 10 Comparison of current density profiles between the 2D (red line) and 3D (blue line) model during the discharge evolution.

Table 3: Computational effort for $2 D$ and $3 D$ simulations

\begin{tabular}{|l|l|l|l|}
\hline Model & DoF & RAM & Time \\
\hline 2D & 36,000 & $300 \mathrm{Mb}$ & $20 \mathrm{~min}$ \\
\hline 3D & $4,000,000$ & $15 \mathrm{~Gb}$ & 1 week \\
\hline
\end{tabular}

\subsection{The effect of plasma spot and pressure inhomogeneities on streamer propagation}

Following the validation of the 3D model, we will now use it to investigate the intrinsic parameters which are responsible for the formation of discharge branching and off-axis propagation. According to the literature at least four causes are involved in streamer branching: Laplacian instabilities [7], pressure instabilities [6], streamer interactions [25] and photoionisation fluctuations [26]. Here we 
consider the effect of plasma spots and low pressure instabilities (bubbles in [6]) on streamer propagation. Plasma spots usually have a spherical form with a radius of several micrometers and a charge density within them that resembles a Gaussian distribution. They represent laser pulses or UV radiation that could be applied in the discharge volume close to the propagating front of the streamer. Additionally, a neutral plasma spot could also describe, in a simplified manner, the interaction of the streamer head with an already formed discharge channel [25]. Plasma spots are introduced in the model as an additional source term in the continuity equations for electrons and ions. Their Gaussian form has a maximum value in the range of $10^{20}-10^{22} \mathrm{~cm}^{-3} \mathrm{~s}^{-1}$ and the spots are applied for a short subnanosecond time interval. In this work the effect of (i) the distance between the plasma spot and the streamer, (ii) the size of the plasma spot and (iii) the charge density in the plasma spot on streamer propagation is considered.

Low pressure instabilities, on the other hand, can appear in the discharge volume in a chaotic way [6]. The magnitude of these fluctuations is dependent on the external conditions of the discharge and is expected to be more significant in the atmosphere (e.g. lightning). In the proposed model they have a spherical spatial form (bubbles) and they are introduced off-axis close to the propagating front. In their volume the ratio E/N is elevated compared to that of ambient air, influencing in this way the transport parameters of the charged species. In this study the relative position of the bubbles and the pressure difference have been studied.

\subsubsection{Plasma spots}

In figure 11 the formation and propagation of a streamer in the presence of a plasma spot is presented. The plasma spot has been placed $100 \mu \mathrm{m}$ from the axis, $110 \mu \mathrm{m}$ away from the streamer head and the maximum charge density production rate is $10^{22} \mathrm{~cm}^{-3} \mathrm{~s}^{-1}$. This instability is applied for 0.1 ns and corresponds to the application of a very fast laser pulse in the discharge volume. Electrons under the influence of the electric field around the streamer head are extracted from the spot, accelerated towards the head and multiplied via ionization collisions. When their multiplication becomes important, Figures 11(b) and 11(c), the propagating front is deformed. A small diagonal subchannel (symbol A in Figure 11(c)) is formed and bridges the gap between the head and the spot. After the formation of the sub-channel, the streamer continues the propagation but off axis, parallel to the initial axis of the discharge (figure 11(d)). The main reason for this pattern of propagation is the strong external geometric field that forces any discharge to eventually move in a direction parallel to the field lines. In order to have diagonal propagation it is necessary to produce seed electrons in the diagonal direction. In an overvolted plane to plane gap the field lines feed seed electrons in directions perpendicular to the electrodes making the off-axis diagonal propagation almost impossible.

It is anticipated that most of the instabilities which arise in such a discharge gap will not be able to produce branching but small changes in the initial direction of the discharge channel. This becomes clear if we compare the evolution of the discharge channel without the addition of the plasma spot and the off axis propagation after introducing the plasma spot, figures 12(a) and 12(b).

Even if the Laplacian instability [7] case is considered, it is questionable that diagonal branching could arise. What could be expected in that case is an initial splitting of the main streamer head, the formation for example of two additional streamer channels, a diagonal propagation of these channels for a while due to the repulsion of the heads and after, as the repulsion reduces with distance, again propagation parallel to the electric field lines. In our opinion the most probable scenario for branching in overvolted parallel gaps is the streamer interactions: reconnection [25] or attraction due to non local photoionisation [11]. Reconnection is caused by electrostatic attraction of a subsequent streamer with a conducting channel left by an early streamer that has already reached the charged electrode with opposite charge and has changed polarity [25].

As Luque shows [11] two streamers of the same polarity do not always repel. At atmospheric pressure and at certain distance away the streamers attract each other: a cloud of electrons is created between the two streamers which makes them coalesce into a single wider one. 
We have performed a parametric analysis on how the magnitude and the position of the several discharge instabilities influence the discharge channel. In Figure 13(a) and 13(b) the influence of a Gaussian plasma spot placed $150 \mu \mathrm{m}$ and $100 \mu \mathrm{m}$ from the axis of symmetry is depicted. In this case the maximum number density of the spot is chosen to be $10^{22} \mathrm{~cm}^{-3} \mathrm{~s}^{-1}$. The role of the distance is quite obvious: the interaction between the streamer head and plasma spot is mainly due to the electric field of the space charge. For distances larger than a critical value, the electron multiplication (in the vicinity of the spot) via ionization collisions is not enough to disturb the propagation path (Figure 13(a)). When the spot is closer, the streamer head deforms thus revealing a tendency for splitting (Figure 13(b)).

Keeping in mind that in order for the streamer channel to be deformed, the distance between the streamer head and plasma spot has to be lower than a critical one, the effect of the magnitude of the plasma Gaussian disturbance placed at $100 \mu \mathrm{m}$ off axis was examined. In the previous cases the maximum density was $A=10^{22} \mathrm{~cm}^{-3} \mathrm{~s}^{-1}$, and in figures $14(\mathrm{a})$ and $14(\mathrm{~b})$ results for $\mathrm{A}=10^{20} \mathrm{~cm}^{-3} \mathrm{~s}^{-1}$ and $\mathrm{A}=10^{22} \mathrm{~cm}^{-3} \mathrm{~s}^{-1}$ are presented respectively. The deformation of the streamer head is again obvious. However, as the maximum charge density of the plasma spot is reduced so is the splitting of the discharge channel, leading to the absence of off - axis propagation when the value is reduced below a critical value.

\subsubsection{Low pressure fluctuations}

The last instability examined deals with low pressure fluctuations that may arise as streamers propagate. In this work a spherical bubble of radius $40 \mu \mathrm{m}$ at a distance of $50 \mu \mathrm{m}$ and 100 $\mu \mathrm{m}$ away from the axis of the main discharge was introduced. Initially the pressure in the bubble was 700 Torr ( $\sim 8 \%$ decrease). The ratio E/N inside the bubble is hence larger than outside. As the streamer head approaches, there is a further increase in $\mathrm{E} / \mathrm{N}$ due to the field enhancement ahead of the avalanche front. As photoionization supplies electrons in the bubble, the elevated $\mathrm{E} / \mathrm{N}$ produces a more rapid rate of ionization than in the rest of the gap. This ionization produces a source of electrons that drift upstream into the gap between the bubble and the approaching avalanche front. The drift velocity of the electrons is also higher in the bubble than outside. As a result, the streamer accelerates into the bubble [6] (see Figure 15). In Figures 15(a), 15(b) and 15(c) the effect on streamer propagation of the bubble pressure and the distance between the bubble and the streamer head is shown. No branching or splitting of the front can be observed in this case. Further calculations have shown that a decrease in the bubble pressure of $\sim 30 \%$ (this is a very extreme case) could deform considerably the shape of the streamer head but cannot produce diagonal streamer branching as can be seen clearly in Figure 15(c).

No streamer branching could be reproduced under these conditions with the application of the 3D model. What were clear and reproducible were the streamer head splitting and the off-axis propagation under specific conditions. It is believed that the small gap length and the electrode configuration plays an important role in the development of streamer branching. Numerical investigations in point to plane geometries as well as longer gaps have to be performed in order to investigate further the development and evolution of branching and the role of Laplacian instabilities. 


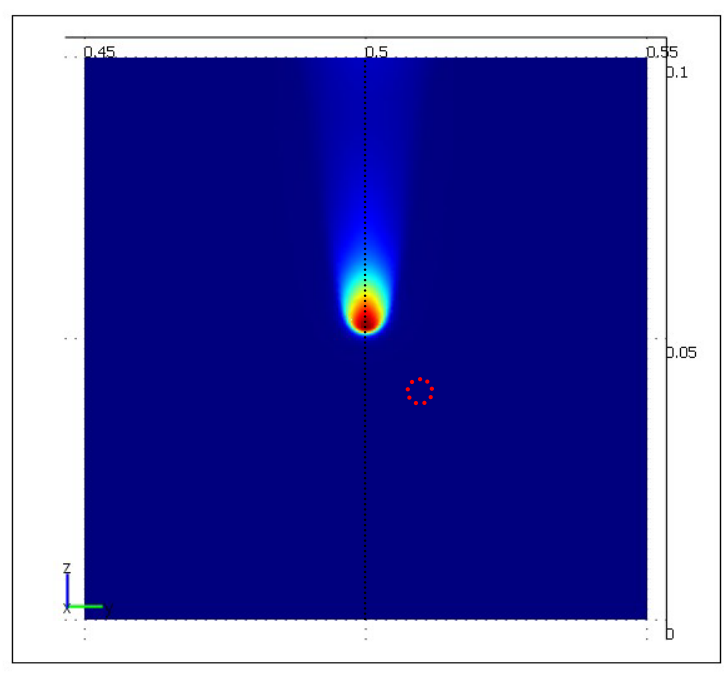

Min: 2946.69

a) Electron density $\left(\mathrm{cm}^{-3}\right)$ at $7.2 \mathrm{~ns}$.

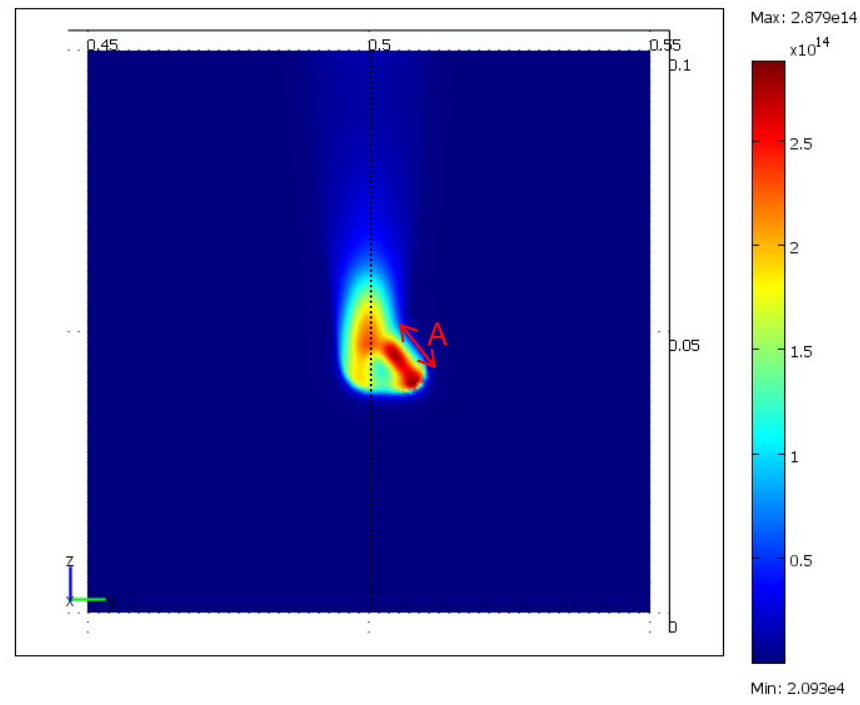

c) Electron density $\left(\mathrm{cm}^{-3}\right)$ at $7.6 \mathrm{~ns}$.

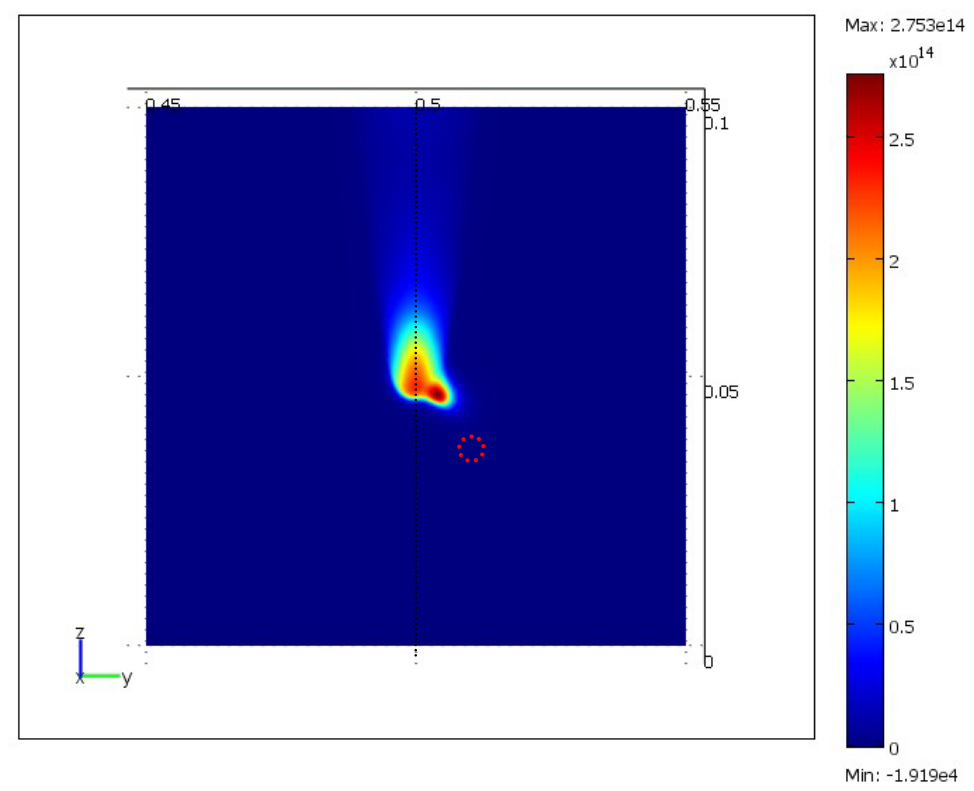

b) Electron density $\left(\mathrm{cm}^{-3}\right)$ at $7.4 \mathrm{~ns}$.

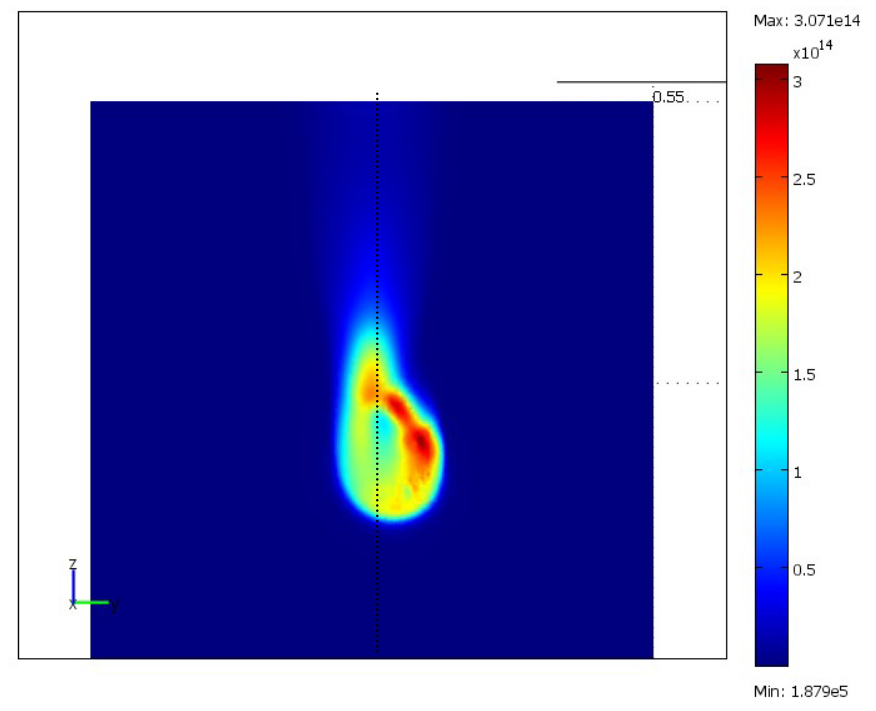

d) Electron density $\left(\mathrm{cm}^{-3}\right)$ at $7.9 \mathrm{~ns}$.

Figure. 11 Electron density evolution during the streamer phase having introduced a plasma spot (red doted circle) off axis close to the streamer head. The maximum charge density production rate of the plasma spot is $10^{22} \mathrm{~cm}^{-3} \mathrm{~s}^{-1}$ and the spot is applied for $0.1 \mathrm{~ns}$. 


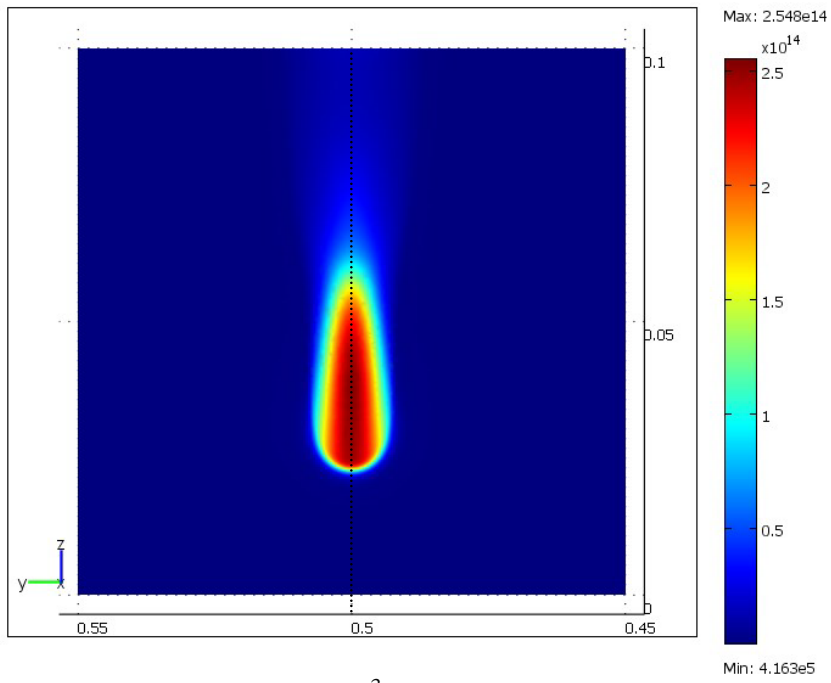

a) Electron density $\left(\mathrm{cm}^{-3}\right)$ at $7.9 \mathrm{~ns}$. No plasma spot present.

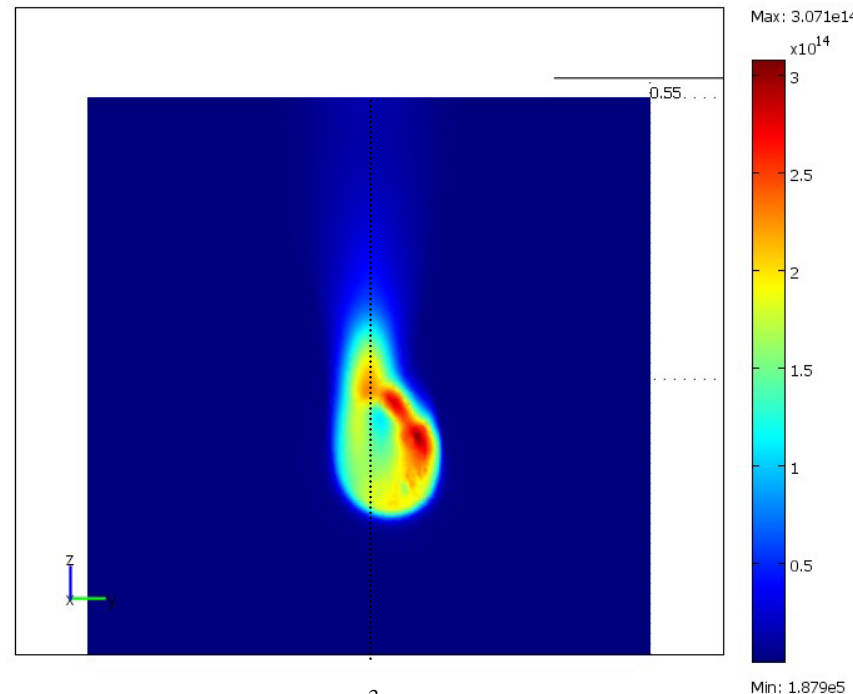

b) Electron density $\left(\mathrm{cm}^{-3}\right)$ at $7.9 \mathrm{~ns}$. Spot at $100 \mu \mathrm{m}$ from the axis of symmetry at the middle of the gap with a maximum charge density production rate $10^{22} \mathrm{~cm}^{-3} \mathrm{~s}^{-1}$.

Figure. 12 Influence of the plasma spot instability on streamer propagation

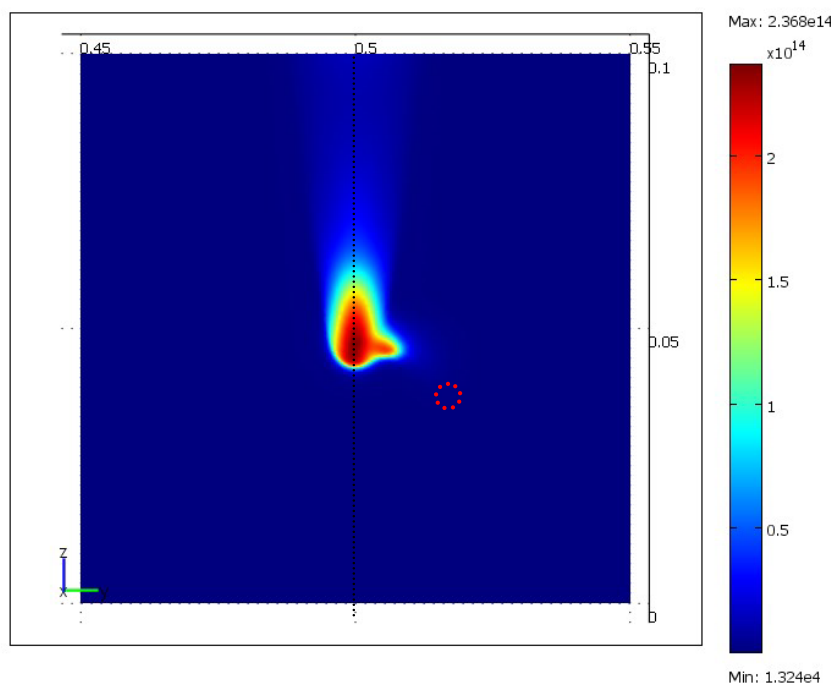

a) Electron density $\left(\mathrm{cm}^{-3}\right)$ at $7.6 \mathrm{~ns}$. Plasma spot at 150 $\mu \mathrm{m}$ from the axis of symmetry.

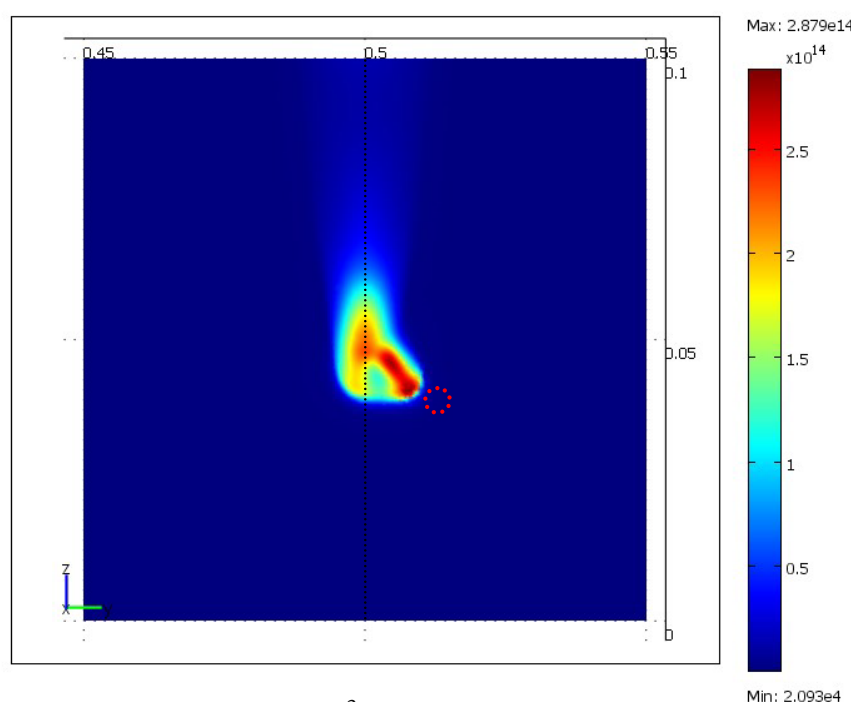

b) Electron density $\left(\mathrm{cm}^{-3}\right)$ at $7.6 \mathrm{~ns}$. Plasma spot at $100 \stackrel{\text { Minn } 2.093 \mathrm{e}}{\mu \mathrm{m}}$ from the axis of symmmetry.

Figure. 13 Influence of the position of the plasma spot instability on streamer propagation. The maximum charge density production rate of the spot is $10^{22} \mathrm{~cm}^{-3} \mathrm{~s}^{-1}$ 


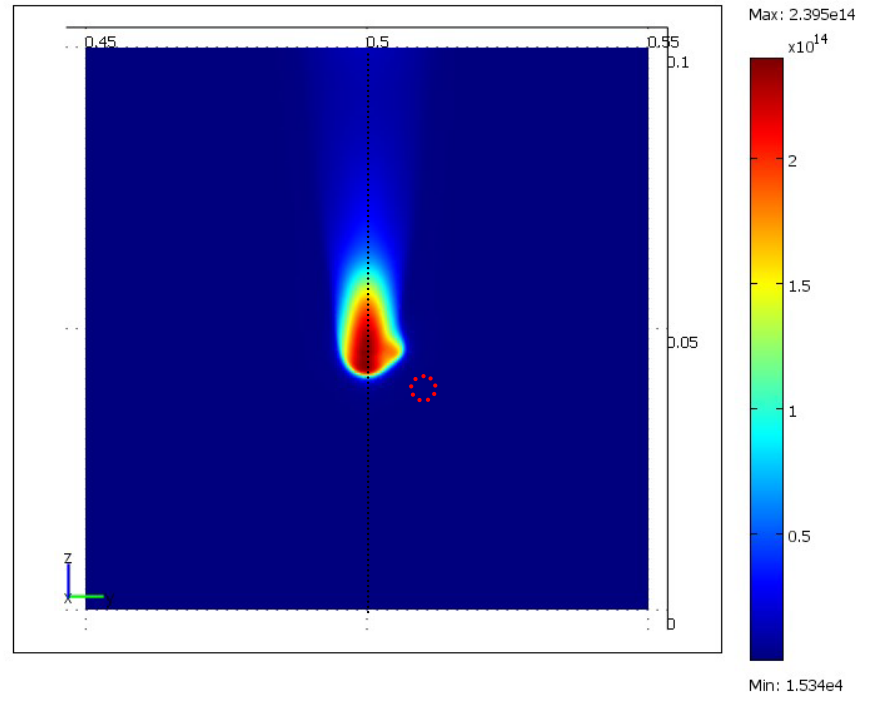

a) Electron density $\left(\mathrm{cm}^{-3}\right)$ at $7.6 \mathrm{~ns}$. The maximum charge density of the spot is $10^{20} \mathrm{~cm}^{-3} \mathrm{~s}^{-1}$.

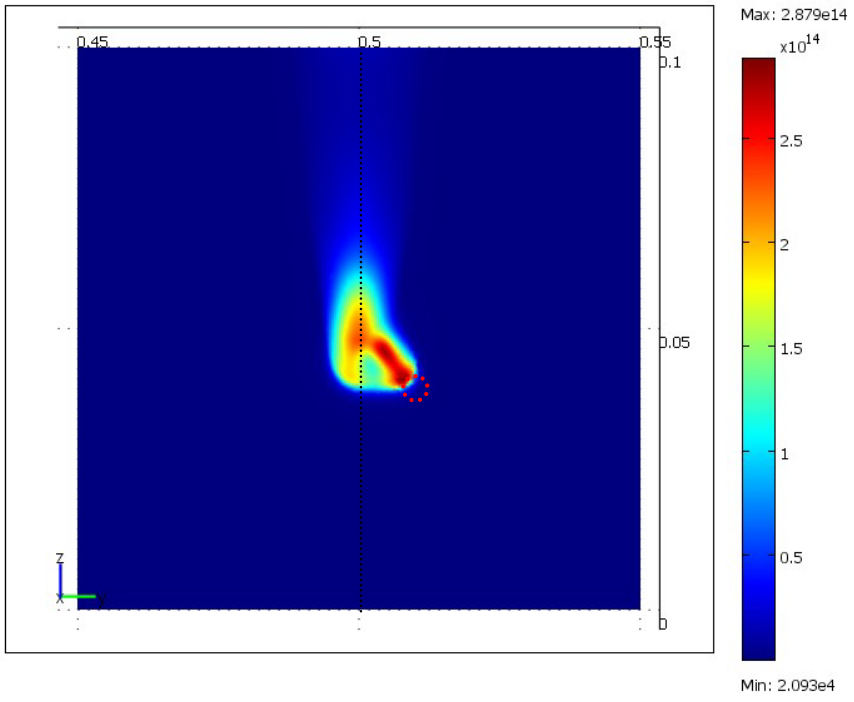

b) a) Electron density $\left(\mathrm{cm}^{-3}\right)$ at $7.6 \mathrm{~ns}$. The maximum charge density of the spot is $10^{22} \mathrm{~cm}^{-3} \mathrm{~s}^{-1}$.

Figure. 14 Influence of the magnitude of the plasma spot instability on streamer propagation. 


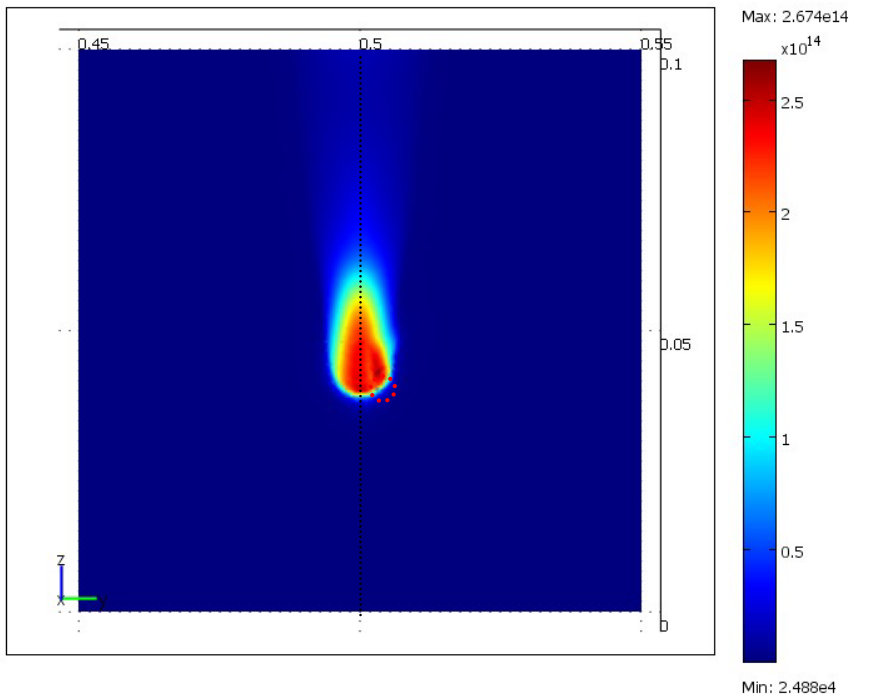

a) Electron density $\left(\mathrm{cm}^{-3}\right)$ at $7.6 \mathrm{~ns}$. Low pressure bubble (700 torr) at $50 \mu \mathrm{m}$ from the axis of symmetry

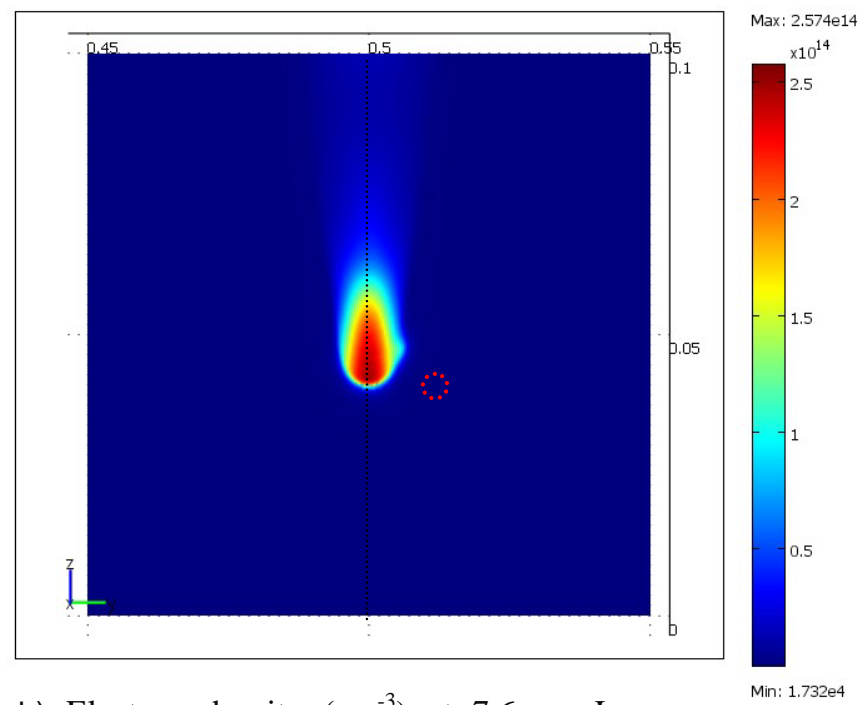

b) Electron density $\left(\mathrm{cm}^{-3}\right)$ at $7.6 \mathrm{~ns}$. Low pressure bubble (700 torr) at $100 \mu \mathrm{m}$ from the axis of symmetry.

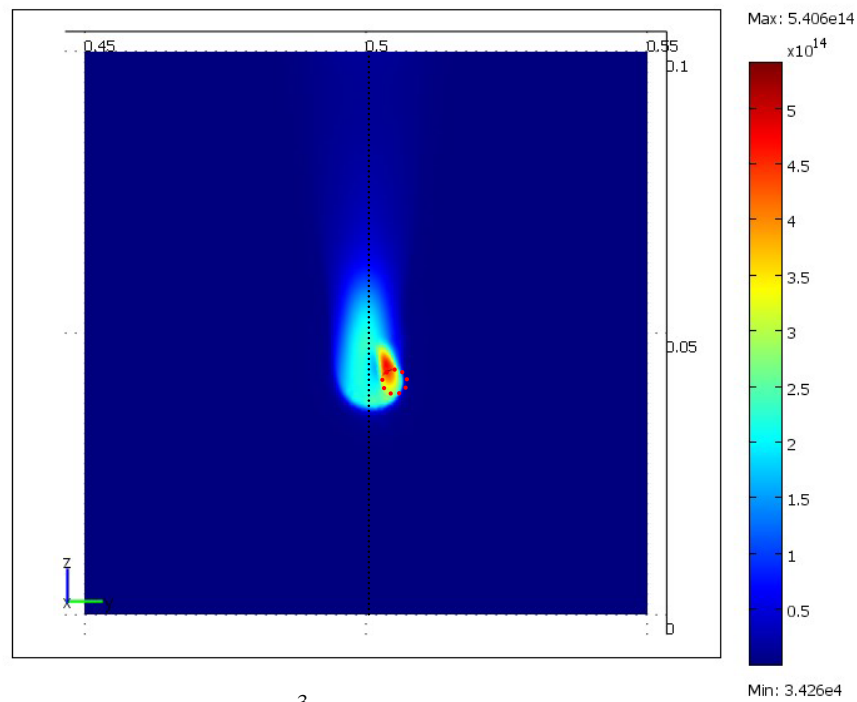

c) Electron density $\left(\mathrm{cm}^{-3}\right)$ at $7.6 \mathrm{~ns}$. Low pressure bubble $(500$ torr) at $50 \mu \mathrm{m}$ from the axis of symmetry

Figure. 15 Influence of the low pressure bubble instability on streamer propagation.

\section{CONCLUSIONS}

Results have been presented from a full three dimensional gas discharge model incorporating photoionisation radiation via the solution of the Helmholtz equations. This has been compared with the two dimensional counterpart. The Helmholtz equations were solved by incorporating the twoexponential fit approach. The avalanche and streamer propagation phase were analysed and a comparison between the 2D axi-symmetric and the 3D model was performed. The two different approaches gave very similar results during the avalanche and streamer phases. The computational cost of the 3D models is very high showing clearly that $2 \mathrm{D}$ models have to be adopted in cases where the cylindrical symmetry is preserved and especially in large gaps. The role of two different discharge instabilities in streamer branching was examined. Plasma spots and low pressure fluctuations during 
the streamer propagation were simulated. Depending on the magnitude and position of the plasma spot, deformations and off-axis propagation of the main discharge channel was observed. Streamer splitting was less sensitive to low pressure discontinuities. Pressure instabilities start to be important when their relative difference to the ambient air is more than $30 \%$. The small gap length and plane to plane electrode configuration are parameters that are believed to limit streamer branching.

\section{ACKNOWLEDGEMENTS}

The authors would like to thank Drs Richard and Vivienne Morrow for some very stimulating technical discussions and editing of the paper.

\section{REFERENCES}

[1] Fridman, G., G. Friedman, A. Gutsol, A. Shekhter, V. Vasilets and A. Fridman, Applied plasma medicine. Plasma Processes and Polymers, 2008. 5(6): p. 503-533.

[2] Boeuf, J., Y. Lagmich, T. Unfer, T. Callegari and L. Pitchford, Electrohydrodynamic force in dielectric barrier discharge plasma actuators. Journal of Physics D: Applied Physics, 2007. 40: p. 652.

[3] Georghiou, G., A. Papadakis, R. Morrow and A. Metaxas, Numerical modelling of atmospheric pressure gas discharges leading to plasma production. Journal of Physics D: Applied Physics, 2005. 38(20): p. R303R328.

[4] Papageorghiou, L., E. Panousis, J. Loiseau, N. Spyrou and B. Held, Two-dimensional modelling of a nitrogen DBD at atmospheric pressure. Journal of Physics D: Applied Physics, 2009. 42: p. 105201.

[5] Georghiou, G., R. Morrow and A. Metaxas, Effect of photoemission on the streamer development and propagation in short uniform gaps. Journal of Physics D: Applied Physics, 2001. 34: p. 200-208.

[6] Babaeva, N. and M. Kushner, Effect of inhomogeneities on streamer propagation: I. Intersection with isolated bubbles and particles. Plasma Sources Science and Technology, 2009. 18: p. 035009.

[7] Ebert, U., C. Montijn, T. Briels, W. Hundsdorfer, B. Meulenbroek, A. Rocco and E. Veldhuizen, The multiscale nature of streamers. Plasma Sources Science and Technology, 2006. 15: p. S118.

[8] Naidis, G., On streamer interaction in a pulsed positive corona discharge. Journal of Physics D: Applied Physics, 1996. 29: p. 779.

[9] Hallac, A., G. Georghiou and A. Metaxas, Three-Dimensional Characterization of Short-Gap Streamers in Air at Atmospheric Pressure. IEEE Transactions on Plasma Science, 2005. 33(2).

[10] Pancheshnyi, S., P. Segur, J. Capeillere and A. Bourdon, Numerical simulation of filamentary discharges with parallel adaptive mesh refinement. Journal of Computational Physics, 2008. 227(13): p. 6574-6590.

[11] Luque, A., U. Ebert and W. Hundsdorfer, Interaction of streamer discharges in air and other oxygen-nitrogen mixtures. Physical review letters, 2008. 101(7): p. 75005.

[12] Kulikovsky, A., Three-dimensional simulation of a positive streamer in air near curved anode. Physics Letters A, 1998. 245(5): p. 445-452.

[13] Luque, A., U. Ebert, C. Montijn and W. Hundsdorfer, Photoionization in negative streamers: Fast computations and two propagation modes. Applied Physics Letters, 2007. 90: p. 081501.

[14] Bourdon, A., V. Pasko, N. Liu, S. C lestin, P. S gur and E. Marode, Efficient models for photoionization produced by non-thermal gas discharges in air based on radiative transfer and the Helmholtz equations. Plasma Sources Science and Technology, 2007. 16: p. 656.

[15] Zheleznyak, M., A. Mnatsakarian and S. Sizyk, Photoionisation of nitrogen and oxygen mixtures by radiation from gas discharges. Teplofizika Vysokikh Temperatur, 1982. 20(423): p. 0.0001.

[16] Kulikovsky, A., The role of photoionization in positive streamer dynamics. Journal of Physics D: Applied Physics, 2000. 33: p. 1514.

[17] Naidis, G., On photoionization produced by discharges in air. Plasma Sources Science and Technology, 2006. 15: p. 253-255.

[18] Kang, W., J. Park, Y. Kim and S. Hong, Numerical study on influences of barrier arrangements on dielectric barrier discharge characteristics. IEEE Transactions on Plasma Science, 2003. 31(4).

[19] Morrow, R. and J. Lowke, Streamer propagation in air. Journal of Physics D: Applied Physics, 1997. 30: p. 614.

[20] Marode, E., D. Djermoune, P. Dessante, C. Deniset, P. Sigur, F. Bastien, A. Bourdon and C. Laux, Physics and applications of atmospheric non-thermal air plasma with reference to environment. Plasma Physics and Controlled Fusion, 2009. 51: p. 124002.

[21] L. Papageorgiou, G.E.Georghiou. and Three dimensional (3D) modeling of a streamer in air at atmosperic pressure incorporating photoionization phenomena. GD conference, 2010.

[22] Kulikovsky, A., Two-dimensional simulation of the positive streamer in N2 between parallel-plate electrodes. Journal of Physics D: Applied Physics, 1995. 28: p. 2483-2493.

[23] Veldhuizen, E., Pulsed positive corona streamer propagation and branching. Journal of Physics D: Applied Physics, 2002. 35: p. 2169.

[24] Sato, N., Discharge current induced by the motion of charged particles. Journal of Physics D: Applied Physics, 1980. 13: p. L3. 
[25] Briels, T., E. Veldhuizen and U. Ebert, Positive streamers in air and nitrogen of varying density: experiments on similarity laws. Journal of Physics D: Applied Physics, 2008. 41: p. 234008.

[26] Nasser, E. and L. Loeb, Impulse streamer branching from Lichtenberg figure studies. Journal of Applied Physics, 1963. 34: p. 3340. 